\title{
Accelerated amyloid deposition, neurofibrillary degeneration and neuronal loss in double mutant APP/tau transgenic mice
}

\author{
Elena M. Ribé, ${ }^{\mathrm{a}}$ Mar Pérez, ${ }^{\mathrm{b}}$ Berta Puig, ${ }^{\mathrm{c}}$ Ignasi Gich, ${ }^{\mathrm{d}}$ Filip Lim, ${ }^{\mathrm{b}}$ Mar Cuadrado, ${ }^{\mathrm{a}}$ \\ Teresa Sesma, ${ }^{a}$ Silvia Catena, ${ }^{a}$ Belén Sánchez, ${ }^{a}$ María Nieto, ${ }^{a}$ Pilar Gómez-Ramos, ${ }^{f}$ \\ M. Asunción Morán, ${ }^{\mathrm{f}}$ Felipe Cabodevilla, ${ }^{\mathrm{a}}$ Lluis Samaranch, ${ }^{\mathrm{a}}$ Lourdes Ortiz, ${ }^{\mathrm{a}}$ Alberto Pérez, ${ }^{\mathrm{a}}$ \\ Isidro Ferrer, ${ }^{\mathrm{c}, \mathrm{e}}$ Jesús Avila, ${ }^{\mathrm{b}}$ and Teresa Gómez-Isla ${ }^{\mathrm{a}, \mathrm{g}, *}$
}

${ }^{a}$ Departmento de Neurología, Universidad de Navarra, Pamplona, Navarra, Spain

${ }^{\mathrm{b}}$ Centro de Biología Molecular Severo Ochoa, Universidad Autónoma de Madrid, Madrid, Spain

${ }^{\mathrm{c} I n s t i t u t ~ d e ~ N e u r o p a t o l o g i a, ~ S e r v e i ~ A n a t o m i a ~ P a t o l o g i c a, ~ H o s p i t a l ~ d e ~ B e l l v i t g e, ~ H o s p i t a l e t ~ d e ~ L l o b r e g a t, ~ S p a i n ~}$

${ }^{\mathrm{d}}$ Departamento de Epidemiología, Hospital Santa Creu i Sant Pau, Barcelona, Spain

${ }^{\mathrm{e}}$ Unitat de Neuropatologia, Experimental, Universitat de Barcelona, Campus de Bellvitge, Hospitalet de Llobregat, Spain

${ }_{\mathrm{f}}^{\mathrm{f}}$ epartamento de Morfología, Facultad de Medicina, Universidad Autónoma de Madrid, Madrid, Spain

${ }^{\mathrm{g}}$ Departamento de Neurología, Hospital Santa Creu i Sant Pau, C/Sant Antoni Ma Claret, 167, Barcelona 08025, Spain

Received 11 March 2005; revised 11 May 2005; accepted 11 May 2005

Available online 24 August 2005

Even though the idea that amyloid $\beta$ peptide accumulation is the primary event in the pathogenesis of Alzheimer's disease has become the leading hypothesis, the causal link between aberrant amyloid precursor protein processing and tau alterations in this type of dementia remains controversial. We further investigated the role of $\beta$-amyloid production/deposition in tau pathology and neuronal cell death in the mouse brain by crossing Tg2576 and VLW lines expressing human mutant amyloid precursor protein and human mutant tau, respectively. The resulting double transgenic mice showed enhanced amyloid deposition accompanied by neurofibrillary degeneration and overt neuronal loss in selectively vulnerable brain limbic areas. These findings challenge the idea that tau pathology in Alzheimer's disease is merely a downstream effect of amyloid production/deposition and suggest that reciprocal interactions between $\beta$-amyloid and tau alterations may take place in vivo.

(C) 2005 Elsevier Inc. All rights reserved.

Keywords: APP; $\beta$-amyloid; Tau; Lisosomes; Stereology; Neuronal loss; Transgenic mice; Alzheimer's disease

\section{Introduction}

In the past decade, the idea that accumulation of amyloid $\beta$ peptide $(A \beta)$ in the brain is the primary influence in Alzheimer's

* Corresponding author. Departmento de Neurología, Hospital Santa Creu i Sant Pau, C/Sant Antoni $M^{\text {a }}$ Claret, 167, Barcelona 08025, Spain. Fax: +3493 2919427/932919275.

E-mail address: mgomezi@hsp.santpau.es (T. Gómez-Isla).

Available online on ScienceDirect (www.sciencedirect.com). disease (AD) pathogenesis has become the leading hypothesis (Hardy and Higgins, 1992; Selkoe, 1991). According to this theory, neurofibrillary tangle (NFT) formation and the loss of neurons and synapses in vulnerable regions that invariably accompanies $A \beta$ deposition in the human $\mathrm{AD}$ brain are said to be downstream events resulting from an imbalance between $A \beta$ production and $\mathrm{A} \beta$ clearance.

The discovery of AD-causing mutations in the amyloid protein precursor (APP) and the presenilins 1 and 2 genes, along with the finding that all of these genetic defects enhance the proteolytic processing of APP to form $A \beta$, is probably the strongest evidence favoring the amyloid cascade hypothesis (reviewed in (Selkoe, 1997)).

The recent finding that active immunization against $\beta$-amyloid is associated with a reduction of amyloid deposits, neuritic pathology and gliotic changes in human $\mathrm{AD}$ brains provides further evidence for the validity of the amyloid cascade hypothesis (Hardy and Selkoe, 2002), even though at this point very important safety issues regarding this therapeutic approach need to be addressed (Schenk, 2002).

A role for $A \beta$ in neurofibrillary degeneration is also supported by recent findings showing that coexpression of human mutant tau and APP in the mouse brain results in increased NFT formation, whereas amyloid deposition remains essentially unaltered (Lewis et al., 2001). Moreover, intracerebral injection of fibrillar A $\beta 42$ in P301L mutant tau transgenic $(\mathrm{Tg})$ mice caused a fivefold increase in the number of NFT, favoring the hypothesis that fibrillar $\mathrm{A} \beta$ can accelerate NFT formation in vivo (Gotz et al., 2001).

All together, the above findings suggest that altered APP processing would precede tau alterations in the pathogenesis of 
AD. Yet, this hypothesis remains controversial mainly because the specific neurotoxic species of $A \beta$ and its effects on neuronal function in vivo have not been defined.

We have had the opportunity to further study the in vivo interactions between $A \beta$ production/deposition and tau phosphorylation/neurofibrillary degeneration in the mouse brain by creating a new double transgenic line termed $\mathrm{APP}^{\mathrm{sw}}$-tau ${ }^{\mathrm{vlw}}$ obtained by crossing Tg2576 and VLW lines expressing human APP harboring the double Swedish mutation (APPswe) and human 4-repeat tau containing a triple mutation (G272V, P301L and R406W), respectively. In brief, $\mathrm{Tg} 2576$ line overexpresses human APP containing the double Swedish mutation K670NM671L in a C57B16jxSJL background. Tg2576 mice develop cerebral $A \beta$ deposits and spatial memory deficits in an agedependent manner but no neurofibrillary tangle formation or significant neuronal loss in CA1 (Hsiao et al., 1996; Irizarry et al., 1997). VLW line overexpresses three tau mutations (G272V, $\mathrm{P} 301 \mathrm{~L}$ and $\mathrm{R} 406 \mathrm{~W}$ ) in a $\mathrm{C} 57 \mathrm{~B} 16 \mathrm{jxCBA}$ background. Immunohistochemical analysis of VLW brains reveals high human mutant tau expression in neuronal cell bodies and neurites in cortex and hippocampus with minimal expression in spinal cord. Ultrastructural analysis shows a pretangle appearance in neurons expressing mutant tau, with tau filaments and increased numbers of lysosomes displaying aberrant morphology similar to that found in human AD brains (Lim et al., 2001). The biochemical study of tau phosphorylation and aggregation patterns in the double mutant $\mathrm{APP}^{\mathrm{sw}}$-tau ${ }^{\mathrm{vlw}}$ line demonstrates an increase in tau phosphorylation at non-proline directed sites and tau aggregation in the double mutant $\mathrm{APP}^{\mathrm{sw}}$-tau ${ }^{\mathrm{vlw}}$ line in comparison to $\mathrm{APP}^{\mathrm{sw}}$ and tau ${ }^{\mathrm{vlw}}$ parental lines, respectively, as we have recently published elsewhere (Perez et al., 2005). Here, we have further characterized the clinicopathological phenotype of this new transgenic mouse model.

\section{Materials and methods}

\section{Transgenic mice}

The progeny obtained by crossing Tg2576 and VLW lines included double mutant $\mathrm{APP} / \mathrm{tau}^{+/+}$(hereafter termed $\mathrm{APP}^{\mathrm{sw}}$ $\left.\operatorname{tau}^{\mathrm{vlw}}\right)$, mutant $\mathrm{APP}^{+/-}\left(\mathrm{APP}^{\mathrm{sw}}\right)$, mutant $\operatorname{tau}^{-/+}\left(\operatorname{tau}^{\mathrm{vlw}}\right)$, and nontransgenic ${ }^{-1-}$ animals.

\section{Spatial reference learning and memory testing (Morris water maze)}

Groups of $10-15 \mathrm{Tg}^{+/+}\left(\mathrm{APP}^{\mathrm{sw}}-\mathrm{tau}^{\mathrm{vlw}}\right), \mathrm{Tg}^{+/-}\left(\mathrm{APP}^{\mathrm{sw}}\right), \mathrm{Tg}^{-/+}$ $\left(\mathrm{tau}^{\mathrm{vlw}}\right)$ and $\mathrm{Tg}^{-/-}$littermates underwent spatial reference learning and memory testing in the Morris watermaze at 9 and 16 months of age (Gómez-Isla et al., 2003). The maze was a circular pool (diameter $1.45 \mathrm{~m}$ ) filled with water at $20^{\circ} \mathrm{C}$. The mice underwent visible-platform training for 3 consecutive days ( 8 trials/day) using a platform raised above the water. This was followed by hiddenplatform training, during which the mice were trained to locate a platform submerged $1 \mathrm{~cm}$ beneath the surface for 9 consecutive days (4 trials/day). Each trial was terminated when the mouse reached the platform or after $60 \mathrm{~s}$, whichever came first. Twentyfour hours after the 12th, 24th and 36th trials, the mice were subjected to a probe trial in which they swam for $60 \mathrm{~s}$ in the pool with no platform. Trials were recorded using an HVS watermaze program for analysis of scape latencies and percent time spent in each quadrant of the pool during probe trials (analysis program Ethovision, Wageningen, The Netherlands).

\section{Neuropathological analysis}

\section{Tissue preparation}

The mice were sacrificed under $\mathrm{CO}_{2}$ and brains were removed and snapfrozen in dry ice. For immunohistochemistry, mice were perfused transcardially with $4 \%$ paraformaldehyde; brains were fixed for $24 \mathrm{~h}$ in $4 \%$ paraformaldehyde in phosphate buffered saline (PBS), $\mathrm{pH} 7.4$, and coronally sectioned at $30 \mu \mathrm{m}$ on a freezing sledge microtome. Groups of $5-7 \mathrm{Tg}^{+/+}\left(\mathrm{APP}^{\mathrm{sw}}-\mathrm{tau}^{\mathrm{vlw}}\right), \mathrm{Tg}^{+/-}$ $\left(\mathrm{APP}^{\mathrm{sw}}\right), \mathrm{Tg}^{-/+}\left(\mathrm{tau}^{\mathrm{vlw}}\right)$ and $\mathrm{Tg}^{-/-}$littermates were studied at 9 and 16 months. Groups of $2-3 \mathrm{Tg}^{+/+}\left(\mathrm{APP}^{\mathrm{sw}}-\mathrm{tau}^{\mathrm{vlw}}\right), \mathrm{Tg}^{-/+}\left(\mathrm{tau}^{\mathrm{vlw}}\right)$ and $\mathrm{Tg}^{-1-}$ littermates were studied at 25 months.

\section{Immunostaining}

$30 \mu \mathrm{m}$ coronal sections were permeabilized with $0.5 \%$ Triton$\mathrm{X} 100$ in PBS, blocked with bovine serum albumin (BSA), and sequentially probed with primary antibody (4G8 and 6E10 mouse anti-A $\beta$ 1:500, Chemicon, Temecula, CA; AT8 mouse anti-human PHF tau 1:50, Innogenetics, Belgium) and the appropriate secondary antibody (anti-mouse IgG1 1:200, Southern Biotechnology, Birmingham, AL; Vector ABC Kit). Sections were processed by Nissl staining for neuronal counts, Thioflavin-T and modified Bielschowsky.

\section{Electron microscopy}

AT-8 immunostained sections were postfixed in $2 \% \mathrm{OsO}_{4}$ for $1 \mathrm{~h}$, embedded in araldite and flat-mounted in formvard-coated slides. After polymerization, selected areas were trimmed, reembedded in araldite, resectioned in ultrathin sections and examined in a $100-\mathrm{kV}$ Jeol electron microscope without heavy metal staining.

\section{Amyloid burden quantification}

Amyloid deposition was quantified using $A \beta$ immunostaining (monoclonal anti-A $\beta$ 4G8 and hrp-anti-mouse) and the anaLYSIS image system (Gomez-Isla et al., 1996; Irizarry et al., 1997). Video images were captured of each region of interest on $30 \mu \mathrm{m}$ sections, and a threshold of optical density was obtained that discriminated staining from background. Manual editing eliminated artefacts. The "amyloid burden", defined as the total percentage of cortex covered by amyloid deposits over three sections, was calculated for CA1, cingulate, dentate gyrus molecular layer, entorhinal cortex (EC), and motor, olfactory and visual cortices.

\section{Stereological neuron counts}

Neuron counts were performed in the CA1 hippocampal subfield, cingulate cortex and EC using the optical dissector technique (West and Gundersen, 1990) in $30 \mu \mathrm{m}$ Nissl stained coronal sections at equally spaced intervals $(450 \mu \mathrm{m})$, excluding neurons in the superficial plane of section. The entire volume of each region of interest was estimated according to the principle of Cavalieri, using the C.A.S.T Grid System (Olympus, Albertslund, Denmark).

The CA1 hippocampal subfield was sampled from its caudal extent anteriorly (Bregma -3.40 to Bregma -1.46) using 50 optical dissectors in each case.

The margins of the lateral EC were defined as follows: caudal, Bregma -3.88; rostral, Bregma -3.16; lateral, rhinal fissure; and 
medial, amygdalopyriform transition area. The number of neurons in the entire region was estimated using 30 optical dissectors in each case.

The cingulate cortex was sampled in a caudal-to-rostral orientation: caudal, section containing the most caudal extent of the dentate gyrus (Bregma -3.64); rostral, extending anteriorly $0.94 \mathrm{~mm}$ (Bregma -2.70); medially, presubiculum; and laterally, visual cortex. The total number of neurons was estimated using 60 optical dissectors in each case.

The average coefficient of error from the sampling technique was $<0.04$ suggesting that a minimal amount of variance observed in the counts is due to variance from the technique.

\section{Statistical analysis}

A mixed multifactorial ANOVA with one within subject factor (day) and three between subject factors (age, gender and transgenic status) for repeated measures was conducted to analyze performances on the Morris watermaze test. Three-way analyses of variance (ANOVA) were carried out to examine main effects and all two-way possible interactions of age, gender and transgenic status on number of neurons and amyloid burden. A Scheffé test was used for post hoc analyses in the absence of interactions.
ANOVA test was used to test for single effects when significant interactions could be demonstrated. In all tests, the level of significance was at $P<0.05$. Data are presented as mean \pm standard error, unless otherwise indicated.

\section{Results}

The levels of mutant APP and tau in APP $^{\mathrm{sw}}-$ tau $^{\mathrm{vlw}}$ adult mice ( 9 months of age) were comparable to APP ${ }^{\mathrm{sw}}$ and VLW parental lines, respectively, as determined by Western blot analysis on brain homogenates (data not shown).

\section{Behavioral testing}

To assess spatial reference learning and memory function, groups of $10-15 \mathrm{Tg}^{+/+}\left(\mathrm{APP}^{\mathrm{sw}}-\mathrm{tau}^{\mathrm{vlw}}\right), \mathrm{Tg}^{+/-}\left(\mathrm{APP}^{\mathrm{sw}}\right), \mathrm{Tg}^{-/+}\left(\mathrm{tau}^{\mathrm{vlw}}\right)$ and $\mathrm{Tg}-/-$ littermates underwent testing in the Morris watermaze at 9 and 16 months of age. All mice were tested in a coded manner.

A mixed multifactorial ANOVA showed no significant interactions or main effects of age or gender but a significant effect of transgenic status on the Morris watermaze test performance (visible platform $P<0.05$, invisible platform $P<0.05$ and probe trials $P<$
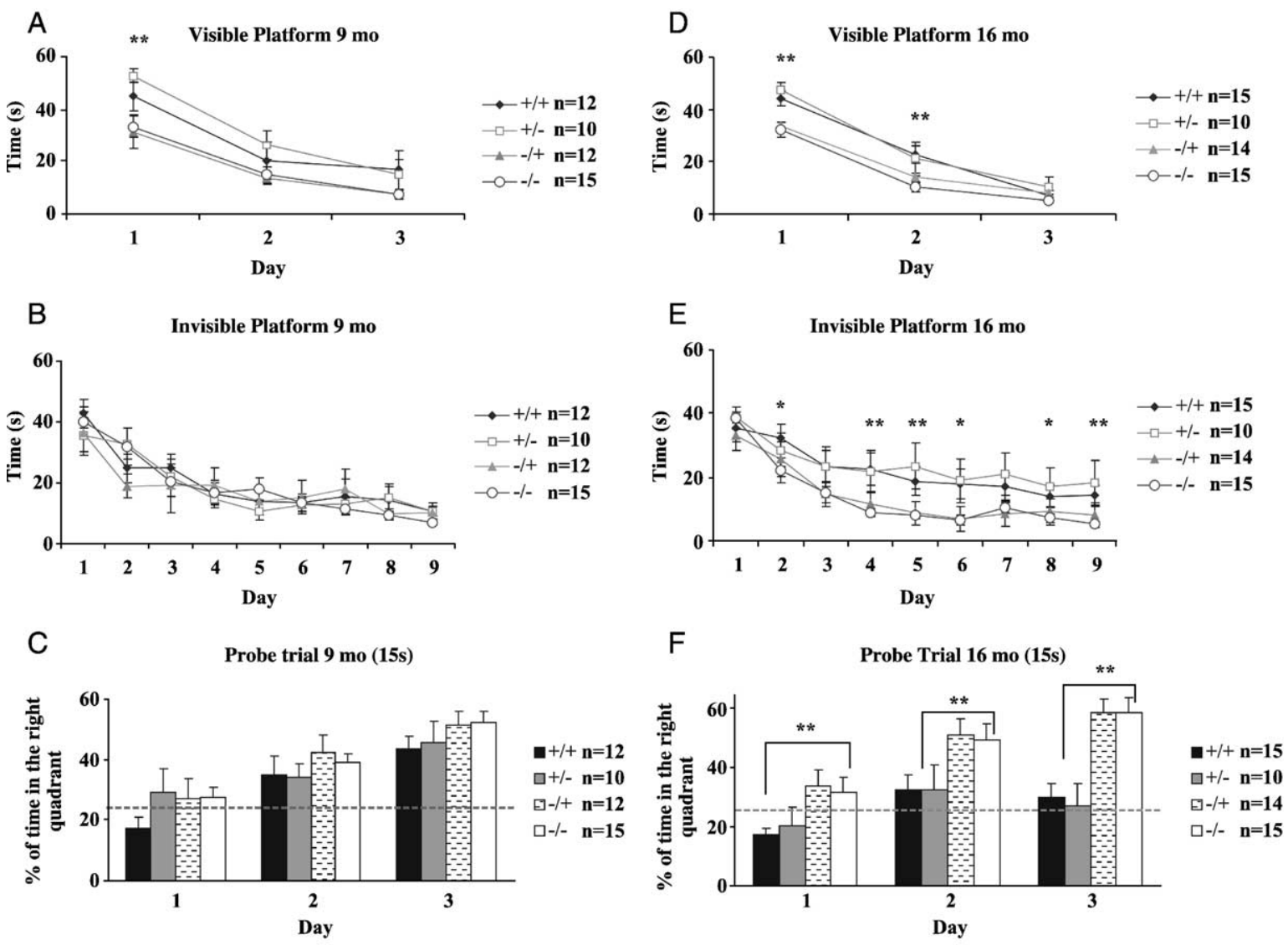

Fig. 1. Morris watermaze performance at $9(\mathrm{~A}-\mathrm{C})$ and 16 months of age (D-F). At 9 months, $\mathrm{APP}^{\mathrm{sw}}$-tau ${ }^{\mathrm{vlw}}$ and $\mathrm{APP}^{\mathrm{sw}}$ mice showed significantly longer scape latencies on day 1 of the visible platform training when compared to tau ${ }^{\text {vlw }}$ or non-transgenic littermate controls (A). No significant differences were detected among groups during any of the 9 days of invisible platform training (B) or the three 15 -s probe trials (C). At 16 months, APP $^{\mathrm{sw}}$-tau ${ }^{\mathrm{vlw}}$ and $\mathrm{APP}{ }^{\mathrm{sw}}$ mice showed significantly longer scape latencies on days 1 and 2 of the visible platform (D), and multiple days of the invisible platform training, when compared to VLW or non-transgenic littermate controls $(\mathrm{E}) . \mathrm{APP}^{\mathrm{sw}}-\mathrm{tau}^{\mathrm{vlw}}$ and $\mathrm{APP}^{\mathrm{sw}}$ mice performed significantly worse than tau ${ }^{\mathrm{vlw}}$ or non-transgenic littermate controls in the three 15-s probe trials $(\mathrm{F}) .{ }^{*} P<0.05, * * P<0.01$. 
0.01). At 9 months of age, a Scheffé post hoc analysis revealed significantly longer scape latencies for $\mathrm{APP}^{\mathrm{sw}}-\mathrm{tau}^{\mathrm{vlw}}$ and $\mathrm{APP}^{\mathrm{sw}}$ mice when compared to tau ${ }^{\mathrm{vlw}}$ and non-transgenic littermates on day 1 of the visible platform training $(P=0.002)$ (Fig. 1A). However, no significant differences could be demonstrated on days $2(P=0.35)$ or $3(P=0.15)$ of the visible platform training, the 9 days of invisible platform training $(P=0.93)$ (Fig. 1B), or the three 60 -s probe trials administered $(P=0.81)$. It has been previously suggested that the sensitivity of this test can be increased by giving shorter probe trials (Gerlai, 2001). Thus, in an attempt to detect subtle spatial memory impairment, we analyzed the performance of mice during the first $15 \mathrm{~s}$ of every probe trial. Again, no significant differences could be demonstrated among genotypes at this age $(P=0.34)$ (Fig. 1C).

At 16 months, a Scheffe test showed that $\mathrm{APP}^{\mathrm{sw}}-\mathrm{tau}^{\mathrm{vlw}}$ and $\mathrm{APP}^{\mathrm{sw}}$ mice performed significantly worse than similarly aged nontransgenic littermate controls with longer scape latencies on multiple days of the visible $(P<0.01)$ (Fig. 1D) and invisible platform training $(P<0.05)$ (Fig. 1E), and spent lower percent of time in the correct quadrant of the pool during the probe trials $(P<0.01)$ (Fig. $1 \mathrm{~F})$. Interestingly, tau ${ }^{\mathrm{vlw}}$ mice did not show impairment in this test up to 16 months when compared to non-transgenic littermate controls, suggesting that mutant tau overexpression in this $\mathrm{Tg}$ line does not in itself cause spatial reference memory alteration.

\section{Neuropathological assessment}

At 9 months, $\mathrm{A} \beta$ immunostaining using the monoclonal antibodies 4G8 and 6E10 demonstrated only scarce amyloid deposits in the $\mathrm{APP}^{\mathrm{sw}}$-tau ${ }^{\text {vlw }}$ brains beginning in the cingulate, motor and entorhinal cortices. The morphology, amount and pattern of regional distribution of the amyloid deposits at this age were comparable to those seen in $\mathrm{APP}^{\mathrm{sw}}$ mice. As expected, no $\mathrm{A} \beta$ immunoreactivity was found in tau ${ }^{\mathrm{vlw}}$ or non-transgenic littermate controls. As with tau ${ }^{\mathrm{vlw}}$ mice, at 9 months $\mathrm{APP}^{\mathrm{sw}}-\mathrm{tau}^{\mathrm{vlw}}$ developed intracytoplasmic straight tau filaments composed of abnormally hyperphosphorylated tau in cortex and hippocampus, becoming consistently present and numerous only in older animals (Lim et al., 2001; Perez et al., 2005). Of note, the ultrastructural study of sarkosyl insoluble tau aggregates from these mice shows that tau filaments are noticeably wider in $\mathrm{APP}^{\mathrm{sw}}-\mathrm{tau}^{\mathrm{vlw}}$ (about $10 \mathrm{~nm}$ in diameter) than in tau ${ }^{\mathrm{VLW}}$ brains (about $2 \mathrm{~nm}$ in diameter), as we have recently published elsewhere (Perez et al., 2005). However, no overt NFT formation could be demonstrated at this age.

Stereologically-based neuron counts were carried out in the CA1 subfield of the hippocampus, cingulate cortex and EC. We concentrated on these regions for neuronal counts because they undergo early amyloid deposition in both transgenic mice and human AD brains and they show marked neuronal loss in humans with AD. A three-way ANOVA showed no significant interactions or main effects of age or gender on total number of neurons but a significant interaction of age and transgenic status on EC and CA1 neuronal counts $(P=0.045$ and 0.025 , respectively). The ANOVA by genotype post hoc analysis at 9 months, showed a significant neuronal loss limited to the EC region of $\mathrm{APP}^{\mathrm{Sw}}-\operatorname{tau}^{\mathrm{vlw}}$ mice by $19 \%$ when compared to the other three groups $(P \leq 0.001)(84,283 \pm$ $4050 \mathrm{APP}^{\mathrm{sw}}-$ tau $^{\mathrm{vlw}}$ vs. $100,644 \pm 4529 \mathrm{APP}^{\mathrm{sw}}, 99,399 \pm 4249$ tau $^{\mathrm{vlw}}$ and 103,606 \pm 3412 non-transgenic littermates) (Fig. 2A). No significant loss of neurons could be demonstrated in any of the regions assessed in $\mathrm{APP}^{\mathrm{sw}}$ or tau ${ }^{\mathrm{vlw}}$ brains compared to similarly aged non-transgenic littermate controls.

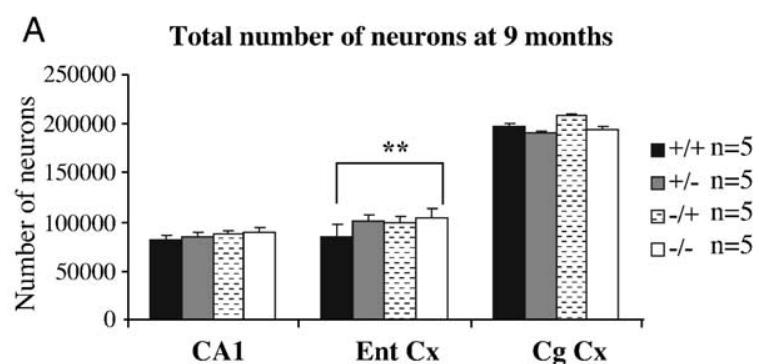

B Total number of neurons at 16 months

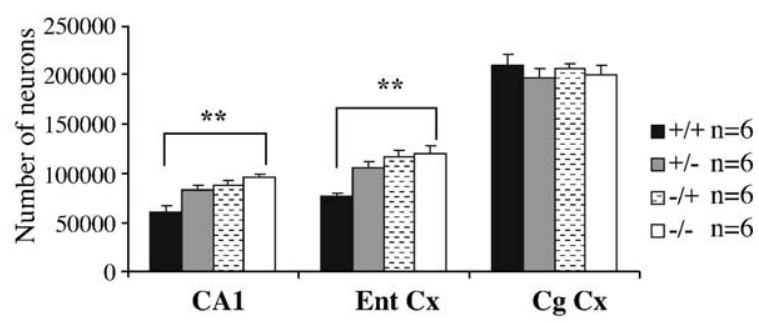

Fig. 2. Stereologically-based neuron counts in the CA1 subfield of the hippocampus, cingulate cortex and EC at 9 (A) and 16 months of age (B). At 9 months, $\mathrm{APP}^{\mathrm{sw}}-$ tau $^{\mathrm{vlw}}$ mice showed significant neuronal loss limited to the EC region by $19 \%$ when compared to the other three groups $(84,283 \pm$ $4050 \mathrm{APP}^{\mathrm{sw}}$-tau ${ }^{\mathrm{vlw}}$ vs. 100,644 $\pm 4529 \mathrm{APP}^{\mathrm{sw}}, 99,399 \pm 4249$ tau $^{\mathrm{VLW}}$ and $103,606 \pm 3412$ non-transgenic littermates). No significant loss of neurons could be demonstrated in any of the regions assessed in $\mathrm{APP}^{\mathrm{sw}}$ or tau ${ }^{\mathrm{VLW}}$ mice in comparison to non-transgenic littermate controls at this same age (A). At 16 months, neuron counts in the EC and CA1 hippocampal region showed a pronounced neuronal loss in $\mathrm{APP}^{\mathrm{sw}}$-tau ${ }^{\mathrm{vlw}}$ brains by $36 \%$ in comparison to the other three groups while no significant neuronal loss could be detected in similarly aged $\mathrm{APP}^{\mathrm{sw}}$ or tau ${ }^{\mathrm{vlw}}$ brains in any of the regions examined $\left(\mathrm{EC}: 76,508 \pm 3798 \mathrm{APP}^{\mathrm{sw}}-\right.$-tau $^{\mathrm{vlw}}$ vs. 105,622 \pm 5927 $\mathrm{APP}^{\mathrm{sw}}, 116,818 \pm 5909 \mathrm{tau}^{\mathrm{vlwC}}$, and $119,769 \pm 7989$ non-transgenic littermates; CA1 hippocampal subfield: $61,135 \pm 5669 \mathrm{APP}^{\mathrm{sw}}-$ tau $^{\mathrm{vlw}}$ vs. $82,842 \pm 4981 \mathrm{APP}^{\mathrm{sw}}, 87,684 \pm 5463 \mathrm{tau}^{\mathrm{vlw}}$ and 95,949 \pm 4103 nontransgenic controls) (B). ${ }^{*} P<0.05,{ }^{* *} P<0.01$.

At 16 months, a two-way ANOVA demonstrated significant main effects of gender $(P<0.03)$ and genotype $(P<0.03)$ as well as an interaction between these two factors $(P<0.04)$ on amyloid deposition in the majority of regions examined. $A \beta$ immunostaining demonstrated up to a fivefold increase in the amount of amyloid deposition in some regions in $\mathrm{APP}^{\mathrm{sw}}$-tau ${ }^{\mathrm{vlw}}$ brains in comparison to similarly aged $\operatorname{Tg} 2576$ mice (Fig. 3A). The difference was statistically significant in every region assessed (ANOVA $P=0.001$ cingulate cortex, $P=0.025$ dentate gyrus molecular layer, $P<0.001$ motor cortex, $P=0.007$ olfactory cortex and $P<0.001$ visual cortex) except for the CA1 hippocampal subfield ( $P=0.336$ ). Interestingly, we observed a very prominent gender difference in the amount of amyloid deposits both in $\mathrm{APP}^{\mathrm{sw}}$ and $\mathrm{APP}^{\mathrm{sw}}-\mathrm{tau}^{\mathrm{vlw}}$ lines. In particular, $\mathrm{APP}^{\mathrm{sw}}-\mathrm{tau}^{\mathrm{vlw}}$ female mice demonstrated significantly higher amyloid burdens in every region examined except for CA1 when compared to $\mathrm{APP}^{\mathrm{sw}}$-tau ${ }^{\text {vlw }}$ males $(P<0.01)$ (Fig. 3B). At this age, many of the amyloid deposits in $\mathrm{APP}^{\mathrm{sw}}$-tau ${ }^{\mathrm{vlw}}$ brains were surrounded by abnormally phosphorylated tau-containing punctate neurites, as demonstrated by AT8 immunostaining, but as for younger mice, no tangle formation could be seen.

At 16 months, neuron counts in the EC and CA1 hippocampal region, but not in the cingulate cortex, showed a 

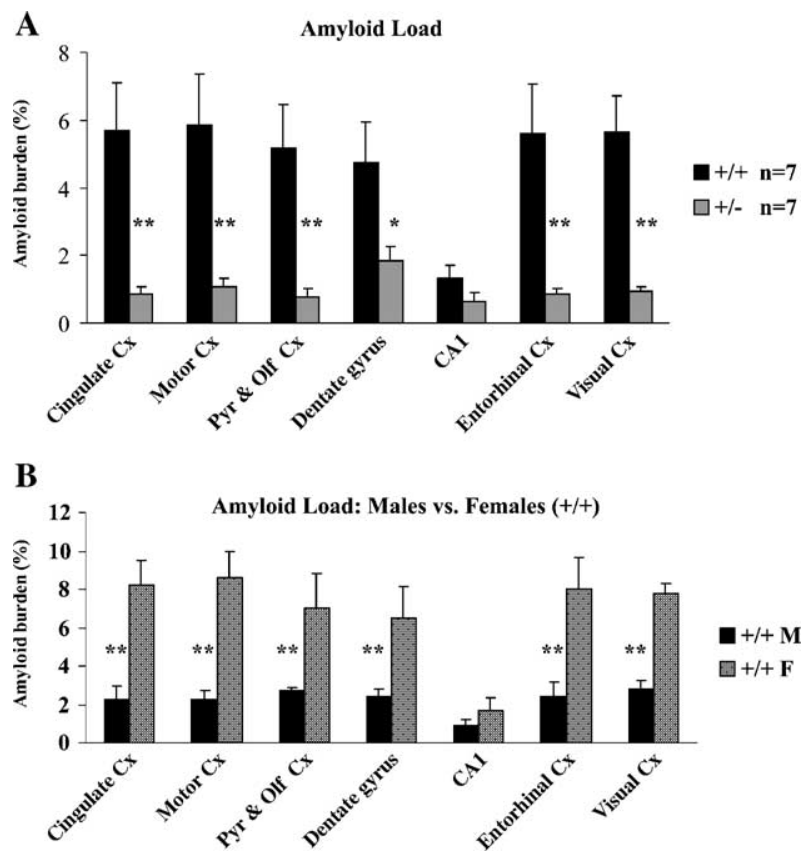

Fig. 3. Amyloid burden quantitation. At 16 months of age, amyloid burden is increased up to fivefold in $\mathrm{APP}^{\mathrm{sw}}$-tau ${ }^{\mathrm{vlw}}$ brains $(N=7,4 \mathrm{~F}$ and $3 \mathrm{M})$ in comparison to similarly aged $\operatorname{APP}^{\mathrm{sw}}$ mice $(N=7,3 \mathrm{~F}$ and $4 \mathrm{M})$. The difference is significant in every region assessed except for the CA1 hippocampal subfield (A). At the same age, $\mathrm{APP}^{\mathrm{sw}}-\mathrm{tau}^{\mathrm{vlw}}$ female mice demonstrated significantly higher amyloid burdens than APP $^{\text {sw }}$-tau ${ }^{\text {vlw }}$ males (B). F: female, M: male. ${ }^{*} P<0.05,{ }^{*} * P<0.01$.

pronounced neuronal loss in $\mathrm{APP}^{\mathrm{sw}}$-tau ${ }^{\mathrm{vlw}}$ brains by $36 \%$ in comparison to similarly aged non-transgenic littermate controls $(P<0.001)$, while no significant neuronal loss could be detected in $\mathrm{APP}^{\mathrm{sw}}$ or tau ${ }^{\mathrm{vlw}}$ brains in any of the regions examined (EC: $76,508 \pm 3798 \mathrm{APP}^{\mathrm{sw}}-$ tau $^{\text {vlw }}$ vs. 105,622 $\pm 5927 \mathrm{APP}^{\mathrm{sw}}, 116,818 \pm$ $5909 \mathrm{tau}^{\mathrm{vlw}}$, and 119,769 \pm 7989 non-transgenic littermates; CA1: $61,135 \pm 5669 \mathrm{APP}^{\mathrm{sw}}$-tau ${ }^{\text {vlw }}$ vs. $82,842 \pm 4981 \mathrm{APP}^{\mathrm{sw}}, 87,684 \pm$ $5463 \mathrm{tau}^{\mathrm{vlw}}$, and 95,949 \pm 4103 non-transgenic controls) (Figs. 2B and 4). In addition, very intense astrocytosis and microgliosis could be seen in areas with abundant amyloid deposits and neuronal loss such as the EC and CA1 in $\mathrm{APP}^{\mathrm{sw}}-$ tau $^{\mathrm{vlw}}$ aged mice. Interestingly, no correlation was found between amyloid burden and the amount of neuronal loss at any of the ages or regions examined (data not shown).

When we examined $\mathrm{APP}^{\mathrm{sw}}$-tau ${ }^{\mathrm{vlw}}$ brains at 25 months, two important features were noted: (1) increased amount of abnormal neurites containing phosphorylated tau in the vicinity of plaques, most of them showing coarse and varicose profiles, and (2) NFT-like structures AT8 and Thioflavin-T positive in the EC region (Fig. 5). Modified Bielschowsky silver stain further demonstrated dystrophic neurites surrounding amyloid plaques and typical NFTs in the EC and, to a lesser degree, in the CA1 area of the hippocampus (Fig. 5). Together, these abnormalities resemble the neuropathological features of senile plaques and NFTs found in conventional AD (Duyckaerts and Dickson, 2003). Electron microscopy studies of AT8 immunostained sections from $\mathrm{APP}^{\mathrm{sw}}-\mathrm{tau}^{\mathrm{vlw}}$ mice at this advanced age showed abundant enlarged degenerating neurites in the periphery of amyloid plaques (Figs. 6A and B). These degenerating neurites were filled with irregular dense bodies that appeared membrane limited in many cases. Some of these enlarged degenerating neurites showed strong AT- 8 immunoreactivity in the irregularly shaped dense bodies as well as in both granular and fibrillar structures present among the dense bodies. Moreover, abundant AT-8 positive neurites filled with immunopositive filaments surrounded the giant amyloid plaques (Figs. 6B-D). In most cases, bundles of amyloid filaments were located adjacent to degenerating neurites filled with dense bodies and also adjacent to
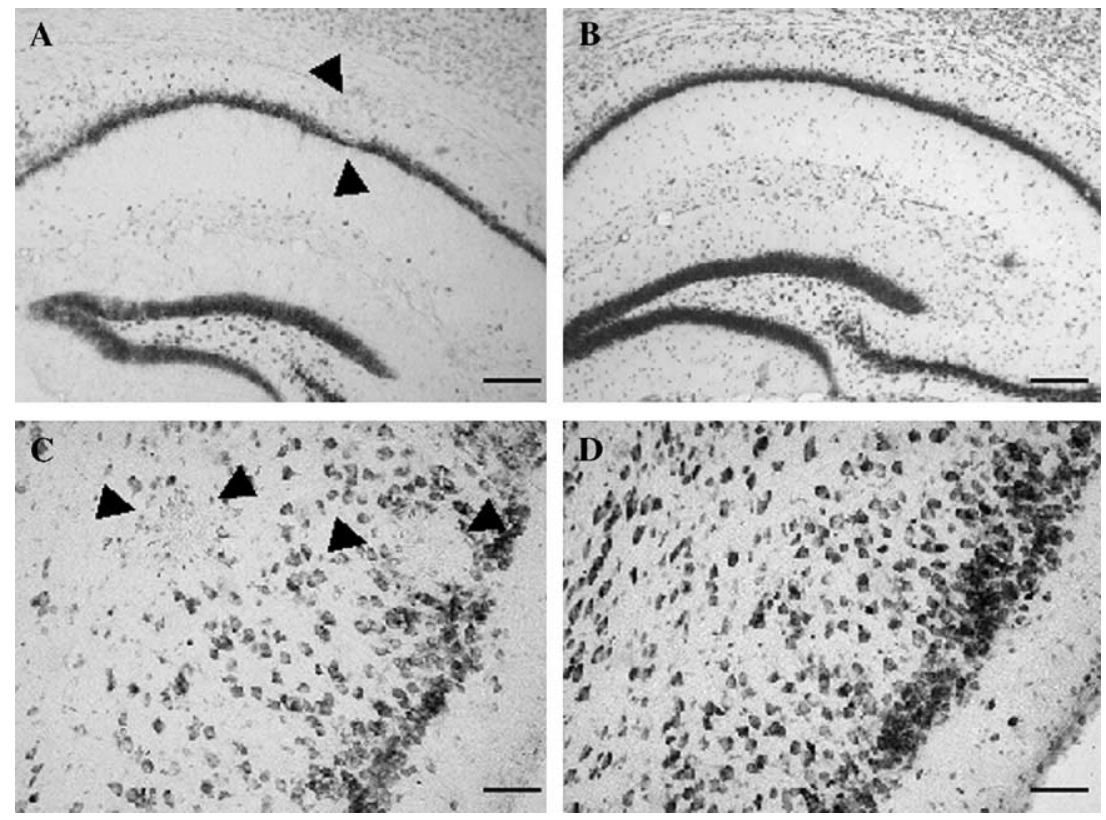

Fig. 4. Photomicrographs of Nissl-stained $30 \mu \mathrm{m}$ thick sections through the hippocampal formation (A and B) and entorhinal cortex (C and D) showing marked loss of neurons in these two regions in an $\mathrm{APP}^{\mathrm{sw}}$-tau $^{\text {vlw }} 16$-month-old mouse (A and C) in comparison to a similarly aged APP ${ }^{\mathrm{sw}}$ littermate (B and D). Arrows indicate areas of massive neuronal depopulation occupied by amyloid deposits. Scale bars A-D, $200 \mu \mathrm{m}$. 


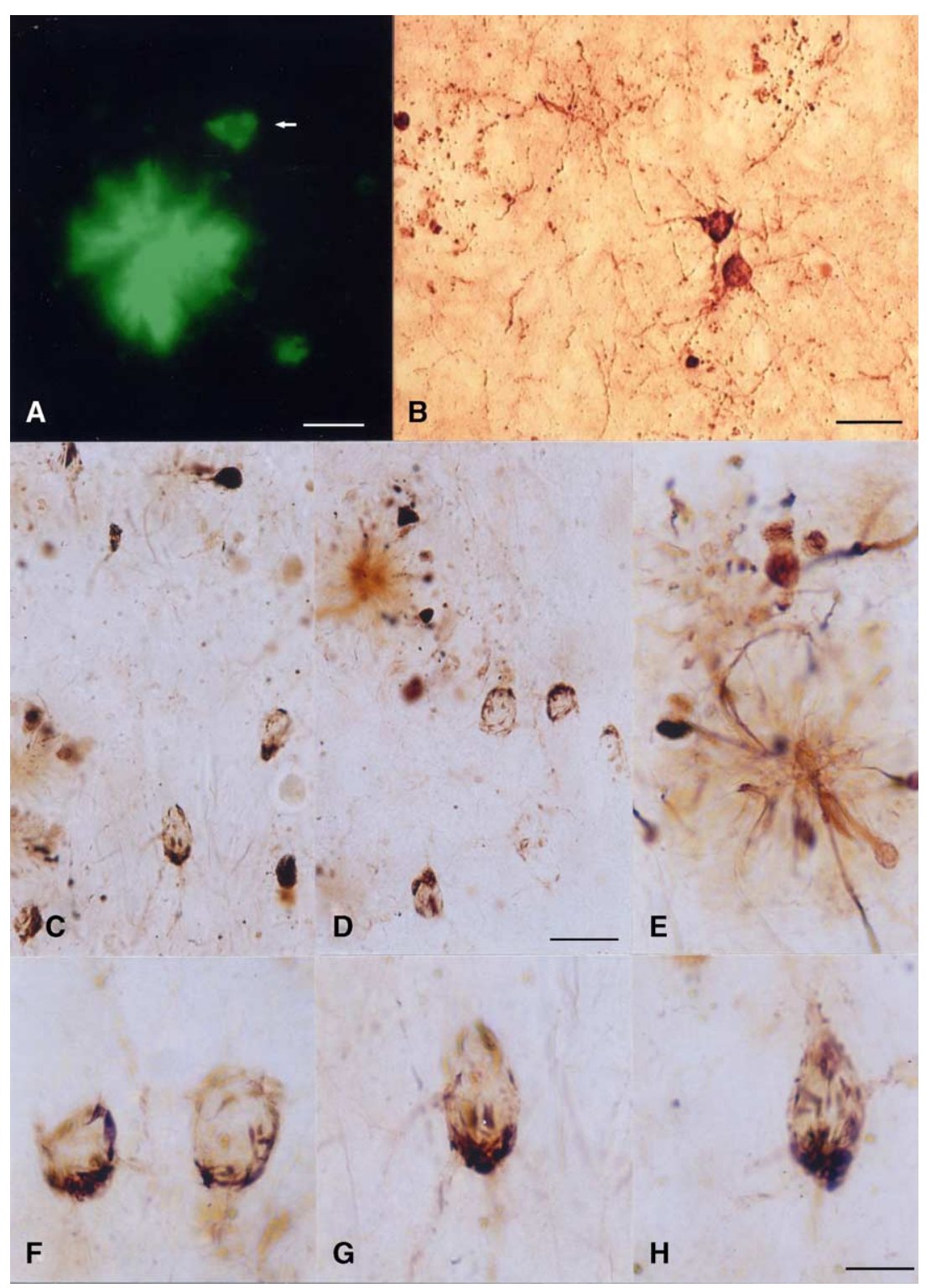

Fig. 5. 25 month-old $\mathrm{APP}^{\mathrm{sw}}$-tau ${ }^{\mathrm{vlw}}$ mice showing thioflavine positive plaques and scarce neurons (arrows) (A), AT8-immunoreactive neurons and cellular processes (B), dystrophic neurites surrounding amyloid cores, and neurons with NFT-like structures stained with the Bielschowsky method (C, D). At high magnification, dystrophic neurites of amyloid plaques show varicose or curb-shaped terminal processes (E) similar to those encountered in human AD brains. Neurons containing NFTs exhibit typical flame-shaped fibrillar inclusions $(\mathrm{F}-\mathrm{H})$ resembling NFTs in human AD. Scale bars, A-20 $\mu \mathrm{m}$; B-40 $\mu \mathrm{m} ; \mathrm{C}-\mathrm{D}$, bar in $\mathrm{D}-25 \mu \mathrm{m}$; $\mathrm{E}-\mathrm{H}$, bar in $\mathrm{H}-10 \mu \mathrm{m}$.

filament filled neurites (Figs. 6C and D). In addition, AT-8-positive filaments were found in apical dendrites of otherwise healthy appearing neurons (Fig. 6A).

\section{Discussion}

The results of this study show that coexpression of human mutant APP and human mutant tau enhances widespread $A \beta$ deposition and triggers neurofibrillary degeneration and neuronal loss in selective vulnerable brain limbic areas in the double mutant $\mathrm{APP}^{\mathrm{sw}}-\mathrm{tau}^{\mathrm{vlw}}$ mouse model.

To date, two other Tg mouse models coexpressing mutant APP and tau have been reported (Lewis et al., 2001; Oddo et al., 2003). Female TAPP mice showed enhanced neurofibrillary pathology in the limbic system and olfactory cortex at 9 to 11 months of age as compared to mice overexpressing mutant tau alone, while the structure and number of amyloid plaques remained comparable to their parental strain $\mathrm{Tg} 2576$ (Lewis et al., 2001). The triple $\mathrm{Tg}$ model published by Oddo and coworkers based on comparable overexpression of mutant human APP, PS1 and tau develops early deficits in long-term synaptic plasticity that correlate with accumulation of intraneuronal $A \beta$ followed by amyloid plaques and NFT formation in an age-dependent fashion (Oddo et al., 2003). Interestingly, extracellular $A \beta$ deposition precedes tau pathology by several months in this latter $\mathrm{Tg}$ line, favoring the amyloid cascade pathogenic hypothesis. 

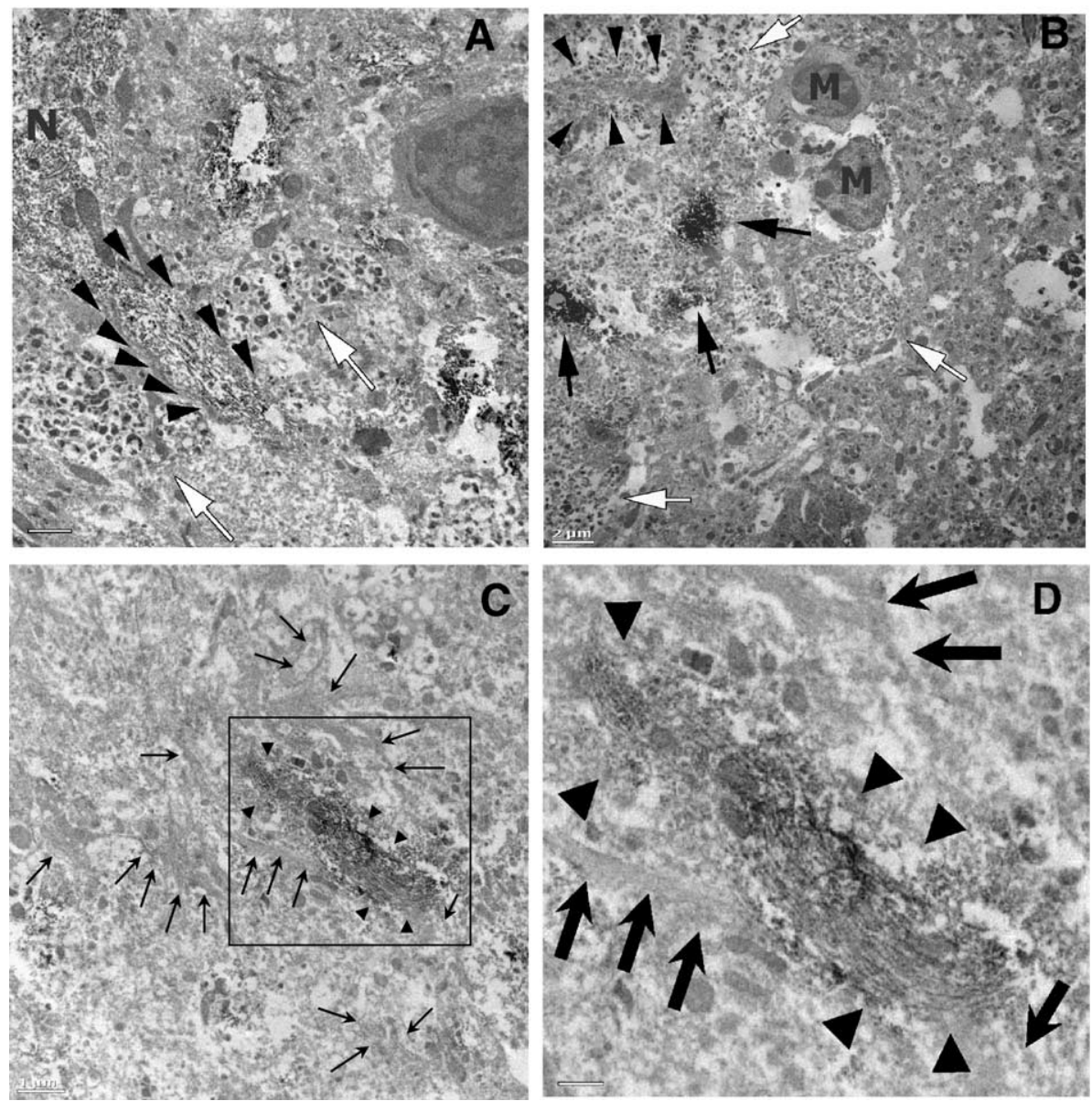

Fig. 6. Electron micrographs from AT-8 immunostained sections of cerebral cortices of 25 month-old APP ${ }^{\mathrm{sw}}$-tau ${ }^{\mathrm{vlw}}$ mice. Apical dendrite of an AT8-positive neurone $(\mathrm{N})$ located at the peripheral area of an amyloid plaque, showing immunoreactive filaments (arrowheads) and surrounded by unlabeled degenerated processes filled with dense bodies (white arrows) (A). Peripheral area of another amyloid plaque with bundles of amyloid fibrils (arrowheads) in the vicinity of both AT8-positive neurites (black arrows) and unlabeled degenerated processes filled with dense bodies (white arrows). M: microglial cells (B). AT8-positive neurite (arrowheads) closely surrounded by abundant bundles of amyloid fibrils (arrows) (C). Higher magnification of the AT8-positive neurite framed in C, showing immunolabeled neurofibrillary degeneration (arrowheads) adjacent to bundles of amyloid filaments (arrows) (D). Calibration bars: $2 \mu \mathrm{m}$ in A and B, 1 $\mu \mathrm{m}$ in $\mathrm{C}$ and $2.6 \mu \mathrm{m}$ in $\mathrm{D}$.

In the present study, we have further characterized a new double Tg model that overexpresses APPsw and a triple FTDP-17 tau mutation $\left(\mathrm{APP}^{\mathrm{sw}}-\mathrm{tau}^{\mathrm{vlw}}\right)$. We have recently presented elsewhere biochemical evidence of enhanced phosphorylation at non-proline directed phosphorylation (NPDP) sites such as serine 262 in $\mathrm{APP}^{\mathrm{sw}}$ tau $^{\text {vlw }}$ mice in comparison to APP ${ }^{\text {sw }}$ mice (Perez et al., 2005). In addition, we observed an increase of the amount of sarkosyl insoluble tau polymers in the double mutants forming tau filaments that are wider in diameter in $\mathrm{APP}^{\mathrm{sw}}$-tau ${ }^{\mathrm{vlw}}$ mice (about $10 \mathrm{~nm}$ ) when compared to tau ${ }^{\mathrm{vlw}}$ mice (about $2 \mathrm{~nm}$ ) (Perez et al., 2005). All together, these findings suggest that $A \beta$ facilitates phosphorylation of tau at NPDP sites and tau polymerization in these mice (Perez et al., 2005). In the present study, the neuropathological assessment showed a significant increase in the amount of amyloid deposition in cortical and limbic areas of $\mathrm{APP}^{\mathrm{sw}}-\mathrm{tau}^{\mathrm{vlw}}$ as compared to $\mathrm{APP}^{\mathrm{sw}}$ mice, and a significant neuronal loss in selective vulnerable areas like the entorhinal cortex and CA1 subfield of the hippocampus, increasing in an age-dependent fashion up to $36 \%$ at 16 months. In addition to enhanced $A \beta$ pathology and cell loss, coexpression of human mutant APP and tau in APP ${ }^{\mathrm{sw}}$-tau ${ }^{\mathrm{vlw}}$ mice not only resulted in increased tau phosphorylation but also triggered NFT-like formation in the EC and CA1 regions at 25 months of age. These results serve to confirm previous observations of enhanced tau pathology in mice coexpressing mutant APP and tau in their brains (Lewis et al., 2001; Oddo et al., 2003). However, the substantial increase of $\mathrm{A} \beta$ pathology observed in $\mathrm{APP}^{\mathrm{sw}}-\mathrm{tau}^{\mathrm{vlw}}$ mice in comparison to their parental line $\mathrm{APP}^{\mathrm{sw}}$ challenges the idea that tau pathology in $\mathrm{AD}$ merely stems from amyloid production/ deposition, and favors a model in which both $\beta$-amyloid and tau pathology can be enhanced by the presence of each other.

The variability noted in the different transgenic mouse models may be influenced by the specific mutation, promoter, expression levels and/or mouse genetic background. In fact, the available data do not differentiate the relative contribution of specific tau mutations to the accelerated amyloid deposition and neuronal loss observed in the $\mathrm{APP}^{\mathrm{sw}}$-tau ${ }^{\text {vlw }}$ brains. The most parsimonious explanation of the accelerated amyloid deposition and cell death seen in the $\mathrm{APP}^{\mathrm{sw}}-\mathrm{tau}^{\mathrm{vlw}}$ mouse model would be that it merely stems from artificially high levels of protein expression.

Interestingly, the amount of $A \beta$ pathology is markedly influenced by mouse gender, with up to fivefold higher amyloid burdens in $\mathrm{APP}^{\mathrm{sw}}$-tau ${ }^{\mathrm{vlw}}$ female mice in comparison to male mice. 
This observation, previously noted by others in the Tg2576 line $\left(\mathrm{APP}^{\mathrm{sw}}\right)$ (Callahan et al., 2001), points to the importance of gender in amyloid production/deposition and highlights the need to take this variable into account in the design of studies carried out on these mice, and possibly on other $\mathrm{Tg}$ mouse models of brain amyloidogenesis (Wang et al., 2003).

The exact mechanisms involved in the accelerated amyloid deposition and neuronal death in the $\mathrm{APP}^{\mathrm{sw}}-$ tau $^{\mathrm{vlw}}$ mouse model need now to be clarified. It has been previously shown that mutant tau overexpression in the $\mathrm{tau}^{\mathrm{vlw}}$ line results in lysosomal abnormalities including increased numbers of lysosomes displaying aberrant morphology (Lim et al., 2001) that mimic those found in human AD brains (Cataldo et al., 1994, 1996). Thus, it is possible that a failure in the endosome-lysosomal machinery could enhance the imbalance between $A \beta$ production and $A \beta$ clearance in $\mathrm{APP}^{\mathrm{sw}}$-tau ${ }^{\mathrm{vlw}}$ mice, accounting, at least in part, for the increase of $A \beta$ pathology observed in their brains. Of note, Vitali et al. (2004) have recently reported significant increases in the amount of soluble $A \beta$ species in brains from patients with FTDP-17 in comparison to frontotemporal dementia lacking distinctive histopathology or normal controls.

Interestingly, cell death in $\mathrm{APP}^{\mathrm{Sw}}-\mathrm{tau}^{\mathrm{vlw}}$ mice preceded overt amyloid plaque formation and NFT formation and did not correlate with amyloid burden in any of the regions examined. Thus, it seems unlikely that neuronal death in the $\mathrm{APP}^{\mathrm{sw}}-\mathrm{tau}^{\mathrm{vlw}}$ line results from enhanced amyloid deposition, although at this point a deleterious effect of soluble or prefibrillar $A \beta$ species on neuron survival in these mice cannot be ruled out.

Learning and memory deficits, as measured by the Morris watermaze test, were quite modest at 9 months of age despite incipient amyloid deposits in $\mathrm{APP}^{\mathrm{sw}}-\mathrm{tau}^{\mathrm{vlw}}$ and $\mathrm{APP}^{\mathrm{sw}}$ brains, and significant neuronal loss in the EC in the former. By 16 months, however, both $\mathrm{APP}^{\mathrm{sw}}$ and $\mathrm{APP}^{\mathrm{sw}}$-tau ${ }^{\mathrm{vlw}}$ mice showed marked spatial reference memory impairment compared to tau ${ }^{\mathrm{vlw}}$ and nontransgenic littermate controls.

Thus, coexpression of human mutant APP and tau in the $\mathrm{APP}^{\mathrm{sw}}$-tau ${ }^{\mathrm{vlw}}$ mouse model enhanced both $\mathrm{A} \beta$ and tau pathology and resulted in significant neuronal loss in selective vulnerable brain areas, providing a new in vivo model that mimics, to a remarkable extent, the human $\mathrm{AD}$ phenotype. We believe this new line of mice represents a very valuable tool for further study of the pathogenetic mechanisms involved in $\mathrm{AD}$ and, more importantly, for testing therapies potentially able to interfere with $A \beta$ and tau pathology and/or to prevent neuronal death. Overall, our results highlight the need for future therapies able to tackle both amyloid production/deposition and tau pathology, in order to successfully halt and/or reverse the pathology and cognitive decline in AD. Supporting this notion, two recent autopsy reports from $A D$ patients who underwent experimental $A \beta$ immunization found a significant decrease in the amount of amyloid deposits in certain brain areas but also showed the presence of additional damage including tau pathology and synapse and neuronal loss that may be irreversible by attacking exclusively $\beta$-amyloid (Ferrer et al., 2004; Nicoll et al., 2003). Keeping in mind that humans and mice differ from each other in many ways, and that some of the results from the presently available animal models for $\mathrm{AD}$ should be interpreted with caution, our results serve to challenge the idea that tau pathology in AD is merely a downstream effect of amyloid production/deposition, and they suggest that reciprocal interactions between $\beta$-amyloid and tau may take place in vivo.

\section{Acknowledgments}

This project was funded in part by EC grant DIADEM QRLT2000-026362, SAF2004-07802 and UTE project CIMA.

\section{References}

Callahan, M.J., Lipinski, W.J., Bian, F., Durham, R.A., Pack, A., Walker, L.C., 2001. Augmented senile plaque load in aged female beta-amyloid precursor protein-transgenic mice. Am. J. Pathol. 158, 1173-1177.

Cataldo, A.M., Hamilton, D.J., Nixon, R.A., 1994. Lysosomal abnormalities in degenerating neurons link neuronal compromise to senile plaques in Alzheimer's disease. Brain Res. 640, 68-80.

Cataldo, A.M., Hamilton, D.J., Barnett, J.L., Paskevich, P.A., Nixon, R.A., 1996. Properties of the endosomal-lysosomal system in the human central nervous system: disturbances mark most neurons in populations at risk to degenerate in Alzheimer's disease. J. Neurosci. 16, 186-199.

Duyckaerts, C., Dickson, D.W., 2003. Neuropathology of Alzheimer's disease. In: D.W. Dickson (Ed.), Neurodegeneration: The Molecular Pathology of Dementia and Movement Disorders, ISN Neuropath Press Basel, pp. 47-65.

Ferrer, I., Boada-Rovira, M., Sanchez-Guerra, M.L., Rey, M.J., Costa-Jussa, F., 2004. Neuropathology and pathogenesis of encephalitis following immunization in Alzheimer's disease. Brain Pathol. 14, 11-20.

Gerlai, R., 2001. Behavioral tests of hippocampal function: simple paradigms complex problems. Behav. Brain Res. 125, 269-277.

Gomez-Isla, T., West, H.L., Rebeck, G.W., Harr, S.D., Growdon, J.H., Locascio, J.J., Perls, T.T., Lipsitz, L.A., Hyman, B.T., 1996. Clinical and pathological correlates of apolipoprotein E epsilon 4 in Alzheimer's disease. Ann. Neurol. 39, 62-70.

Gómez-Isla, T., Irizarry, M.C., Mariash, A., Cheung, B., Soto, O., Schrump, S., Sondel, J., Kotilinek, L., Day, J., Schwarzschild, M.A., Cha, J.H., Newell, K., Miller, D.W., Ueda, K., Young, A.B., Hyman, B.T., Ashe, K.H., 2003. Motor dysfunction and gliosis with preserved dopaminergic markers in human alpha-synuclein A30P transgenic mice. Neurobiol. Aging 24, 245-258

Gotz, J., Chen, F., van Dorpe, J., Nitsch, R.M., 2001. Formation of neurofibrillary tangles in P3011 tau transgenic mice induced by Abeta 42 fibrils. Science 293, 1491-1495.

Hardy, J.A., Higgins, G.A., 1992. Alzheimer's disease: the amyloid cascade hypothesis. Science 256, 184-185.

Hardy, J.A., Selkoe, D.J., 2002. The amyloid hypothesis of Alzheimer's disease: progress and problems on the road to therapeutics. Science 297, $353-356$

Hsiao, K., Chapman, P., Nilsen, S., Eckman, C., Harigaya, Y., Younkin, S., Yang, F., Cole, G., 1996. Correlative memory deficits, Abeta elevation, and amyloid plaques in transgenic mice. Science 274, 99-102.

Irizarry, M.C., McNamara, M., Fedorchak, K., Hsiao, K., Hyman, B.T., 1997. APPSw transgenic mice develop age-related A beta deposits and neuropil abnormalities, but no neuronal loss in CA1. J. Neuropathol. Exp. Neurol. 56, 965-973.

Lewis, J., Dickson, D.W., Lin, W.L., Chisholm, L., Corral, A., Jones, G., Yen, S.H., Sahara, N., Skipper, L., Yager, D., Eckman, C., Hardy, J., Hutton, M., McGowan, E., 2001. Enhanced neurofibrillary degeneration in transgenic mice expressing mutant tau and APP. Science 293, $1487-1491$.

Lim, F., Hernandez, F., Lucas, J.J., Gomez-Ramos, P., Moran, M.A., Avila, J., 2001. FTDP-17 mutations in tau transgenic mice provoke lysosomal abnormalities and tau filaments in forebrain. Mol. Cell. Neurosci. 18, $702-714$.

Nicoll, J.A.R., Wilkinson, D., Holmes, C., Steart, P., Markham, H., Weller, R.O., 2003. Neuropathology of human Alzheimer disease after immunization with amyloid-peptide: a case report. Nat. Med. 9, $448-452$ 
Oddo, S., Caccamo, A., Shepherd, J.D., Murphy, M.P., Golde, T.E., Kayed, R., Metherate, R., Mattson, M.P., Akbari, Y., LaFerla, F.M., 2003. Tripletransgenic model of Alzheimer's disease with plaques and tangles: intracellular Abeta and synaptic dysfunction. Neuron 39, 409-421.

Perez, M., Ribe, E., Rubio, A., Lim, F., Moran, M.A., Ramos, P.G., Ferrer, I., Isla, M.T., Avila, J., 2005. Characterization of a double (amyloid precursor protein-tau) transgenic: tau phosphorylation and aggregation. Neuroscience 130, 339-347.

Schenk, D., 2002. Amyloid-beta immunotherapy for Alzheimer's disease: the end of the beginning. Nat. Rev., Neurosci. 3, 824-828.

Selkoe, D.J., 1991. The molecular pathology of Alzheimer's disease. Neuron 6, 487-498.
Selkoe, D.J., 1997. Alzheimer's disease: genotypes, phenotypes, and treatments. Science 275, 630-631.

Vitali, A., Piccini, A., Borghi, R., Fornaro, P., Siedlak, S.L., Smith, M.A., Gambetti, P., Ghetti, B., Tabaton, M., 2004. Soluble amyloid betaprotein is increased in frontotemporal dementia with tau gene mutations. J. Alzheimer's Dis. 6, 45-51.

Wang, T., Tanila, H., Puolivali, J., Kadish, I., van Groen, T., 2003. Gender differences in the amount and deposition of amyloid beta in APPswe and PS1 double transgenic mice. Neurobiol. Dis. 14, 318-327.

West, M.J., Gundersen, H.J., 1990. Unbiased stereological estimation of the number of neurons in the human hippocampus. J. Comp. Neurol. 296, $1-22$. 